\title{
Design Verification of Web Applications Using Symbolic Model Checking
}

\author{
Eugenio Di Sciascio ${ }^{1}$, Francesco M. Donini ${ }^{2}$, Marina Mongiello ${ }^{1}$, \\ Rodolfo Totaro $^{1}$, and Daniela Castelluccia ${ }^{1}$ \\ 1 Dipartimento di Elettrotecnica ed Elettronica \\ Politecnico di Bari, Italy \\ \{disciascio,mongiello,r.totaro\}@poliba.it \\ ${ }^{2}$ Università della Tuscia Viterbo, Italy \\ donini@unitus.it
}

\begin{abstract}
Fast and reliable development of Web Applications (WA) calls for methods that address systematic design, and tools that cover all the aspects of the design process and complement the current implementation technologies. To ensure the reliability of WA it is important that they be validated and verified at early design phase. We focus on black-box, automated verification of the UML design of a WA using Model Checking techniques.
\end{abstract}

\section{Introduction}

Web Applications (WA) are a class of software systems that support a wide range of important activities, ranging from business functions to scientific and medical applications. The evolution speed of such applications makes Web Engineering a complex activity whose strategies are still being developed. The development of WA needs both methods and formalisms that address systematic design, and tools that can cover all the aspects of the design process and complement the current implementation technologies. Given the relevance of the activities performed by WA, it is important to ensure their reliability through a validation and verification process. Particularly, we consider the design phase and propose a method for checking the correctness of the UML design.

We choose for our purpose the Model Checking method [1] a technique for sound and complete reasoning about finite-state transition systems, that performs an automated verification of a system model with respect to its specification. Specifications are expressed in a logical formalism, generally a logic within a temporal framework.

The main advantage of model checking is that it can be performed automatically unlike test and other formal methods that need user interaction.

Several verification tools have been developed for system analysis based on different formal models. In our proposal we use Symbolic Model Verifier (SMV )[10].

First of all, we propose a mathematical model of a WA partitioning the usual Kripke structure into windows, links, pages and actions. Then we specify properties to be checked in a temporal logic, Computation Tree Logic (CTL). Verification is performed adapting the SMV model checker to our formalism.

An implemented system embeds a parser to perform the automated parsing of the XMI output of the UML tool and to automatically build the SMV model to be verified 
with respect to specifications. The remaining of the paper is organized as follows: in Section 2 we describe the model we propose for web applications and the properties to be verified. Section 3 describes the evaluation environment and the implemented system. In the last Section we describe some related works and the conclusion.

\section{Proposed Model}

The complexity of the hypertextual structure of the Web cannot be modelled using a simple graph structure in which nodes represent pages and arcs represent hyperlinks. In fact, the widespread use of frames, while controversial, makes a window be composed by several pages. Moreover, new implementation technologies such as scripts, servlets, applets add dynamic properties to web pages. Hence, links can lead to a new window or start an action inside a dynamic page. It is required a more compact and powerful model to convey the complexity of the linked page, the hierarchy of windows, the type of different media linked to web pages, the actions that can be performed.

We propose a mathematical model of a WA based on an extension of the simple graph generally adopted to model the pages and the links between pages. The main advantage of the model is that it is also a support for the formal verification of a WA properties. In previous papers [13], [12] we proposed a model for automatic check of web applications. Here we extend the model with the possibility to represent actions performed in a page.

More specifically, we propose an extension of the Kripke structure generally used to convey the semantics of CTL. The model is translated in a proper CTL model. States in the model are windows, pages, links and actions since a state in the model represents everything is visible in an observation.

Definition 1. A Web Application Graph $(W A G)$ is a graph $G=(N, C)$ where nodes $N$ are divided as $N=W \cup P \cup L \cup A$ (Windows, Pages, Links and Actions), such that

1. $W, P, L, A$ are pairwise disjoint, i.e. $W \cap P=\emptyset, W \cap L=\emptyset W \cap A=\emptyset L \cap P=\emptyset$ $L \cap A=\emptyset P \cap A=\emptyset$ and

2. arcs connect only windows with pages, pages with links or actions, links with windows and actions with windows, i.e. $C \subseteq(W \times P) \cup(P \times(L \cup A)) \cup((L \cup A) \times W)$;

3. $\forall w \in W \exists p \in P:(w, p) \in C$ "Every window contains at least one page";

4. $\forall x \in(L \cup A) \exists w \in W:(x, w) \in C$ "Every link points to a window and every action creates a window".

Definition 2. A navigation path is a sequence $w_{1} w_{2} \ldots w_{n}$ where $\forall 1<i<n-1$

$$
\exists p \in P \exists x \in(L \cup A): w_{i} \rightarrow p \wedge p \rightarrow x \wedge x \rightarrow w_{i+1}
$$

Modeling a WAG in CTL Computation Tree Logic can be used to express and verify properties of the above Web Application Graph, if nodes of the graph are taken as states and arcs as state transitions. It is sufficient to reserve four propositional letters $w, p, l, a$ to distinguish nodes modeling windows, pages, links and actions respectively. Then a correct translator will assign exactly one letter among $w, p, l, a$ to each state, and enforce that transitions occur only from windows to pages they contain, from pages to 
links they contain or actions they perform, and from links or actions to the next window. Incidentally, we note that such conditions could also be verified in the WAG by checking the following CTL formulas (where numbers correspond with those in Definition 1):

$$
\begin{aligned}
- & A G((w \vee p \vee l \vee a) \wedge(\neg w \vee \neg p) \wedge(\neg w \vee \neg l) \wedge(\neg w \vee \neg a) \wedge(\neg p \vee \neg a) \wedge \\
& \wedge(\neg p \vee \neg l) \wedge(\neg p \vee \neg a) \wedge(\neg l \vee \neg a)) \\
- & A G(w \Rightarrow A X p \wedge p \Rightarrow A X(l \vee a) \wedge l \Rightarrow A X w \wedge a \Rightarrow A X w)
\end{aligned}
$$

We stress the fact that the original transitions in the WA are from a window to another window, and these transitions are kept in our state model. The transitions from a window to pages they contain, and from pages to links and actions pages contain, are only a technical way to model frames and security properties.

Many interesting properties can be checked if other propositional letters are used to capture the relevant content of windows, pages, links or action. For instance we can introduce the following letters: 1) private denotes that a window or a page contains private information; 2) login, logout denote that an action is a login or a logout action; 3) error denotes that a page contains an error message.

In our model we have to check that these letters are used correctly with the following CTL specification:

1. private is applicable only to pages or windows, so it is not applicable to links or actions: $A G(l \vee a \Rightarrow \neg$ private $)$

2. login and logout are applicable only to actions: $A G(w \vee p \vee l \Rightarrow \neg \operatorname{login} \wedge \neg \operatorname{logout})$

3. error is applicable only to pages: $A G(w \vee l \vee a \Rightarrow \neg$ error $)$

4. a private window must contain at least one private page:

$A G(w \wedge$ private $\Rightarrow E X($ private $))$

5. a not private window must not contain private pages:

$A G(w \wedge \neg$ private $\Rightarrow A X(\neg$ private $))$

Using these propositions we can check some interesting properties of a web application design. For example we can check whether the access to private page occurs through a login, hence whether it is correct:

6. we must find some private information after a login action: $A G(\operatorname{login} \Rightarrow E F($ private $))$

7. after a login action we can make a logout action in the future or the application must manage a login error and it must be possible to make a login again: $A G(\operatorname{login} \Rightarrow$ $A G(w \Rightarrow E X((E X$ logout $) \vee$ error $) \vee E F l o g i n)$

8. after a logout action we can load only not private pages before a new login: $A G($ logout $\Rightarrow A(\neg$ privateUlogin $))$

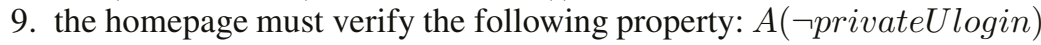

Another property of web application design concerns the error management; we can check the web application behavior when an error occurs. For instance:

10. for every not logout action the web application must manage eventually an error page: $A G(a \wedge \neg$ logout $\Rightarrow E X E X$ error $))$

11. the user must repeat the login action when an error occurs: $A G($ error $\Rightarrow A(\neg$ privateUlogin $))$ 


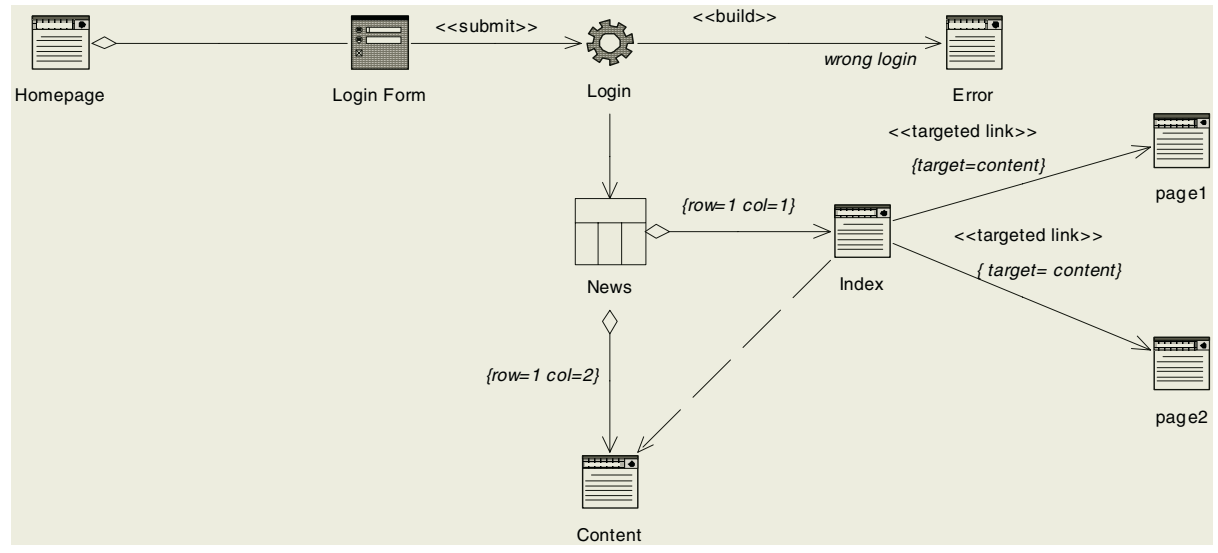

Fig. 1. UML model of the checked web application design

Definition 3 (Verifying a Web Application). Given a WAG $G$ modeling a web application, an initial state $s$ and a property $p$, the web application verifies $p$ iff $p$ holds for $s$ in $G$.

A deployed implementation of our approach will embed inside an automatic verifier for CTL ; however, for building a prototype showing the feasibility of the approach, the verification phase may be also performed using an available tool, such as SMV . In this case, the verification process consists in expressing the Web Application Graph in the SMV input language - also with the help of parametric modules - and then launch the verification.

\section{Evaluation Environment}

The method we propose is made up of two phases: the fist one is the check of a web application during the design phase based on its UML model. In a second step the check will be extended to the web application implementation.

In the first step, we use the UML design of the application developed according to the methodologies proposed by Conallen [2]. In the UML diagram, the components of an application are labelled with the proper properties, e.g. login, logout, private, error in order to perform the translation in the SMV model. An implemented system embeds the SMV verifier to check the model with respect to the specifications described in Section 2.

Our system automatically translates the output (in XMI format) of the UML tool used for the design in the SMV code that models the corresponding WAG. To show the rationale of the approach, let us consider the UML design model in Figure 1. Figure 2 shows the corresponding WAG, in which the dotted lines represent the modifications on the model after verification.

The model was checked against the specifications described in Section 2. Verification found several faults in the model. First of all, in the WAG did not exist a state 


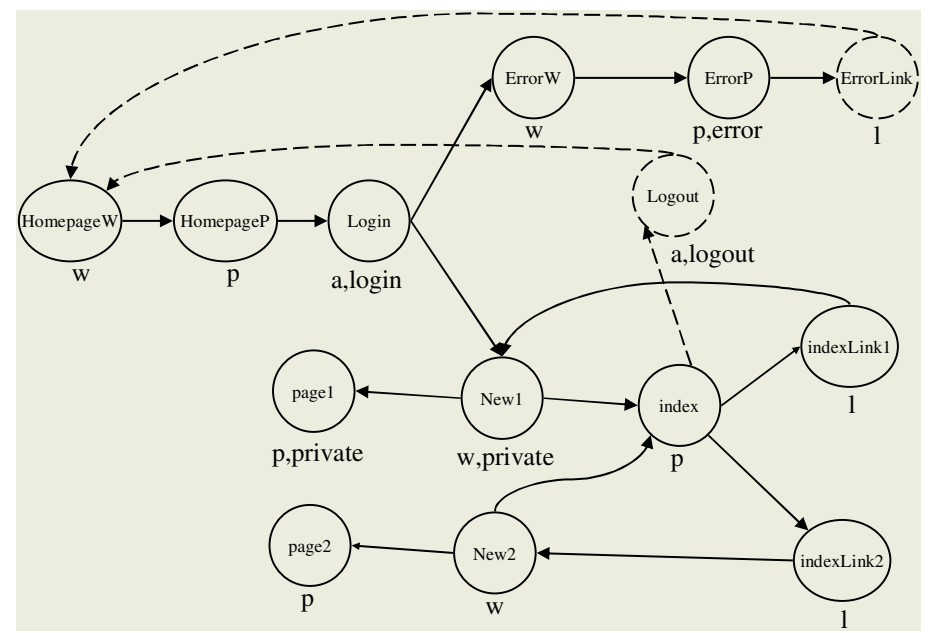

Fig. 2. WAG corresponding to the UML model shown in Figure 1

labelled with the private property, hence the property 6 in previous section was not satisfied. Besides, after a login it should be possible to perform a logout action, the model checker verified the absence of a logout state in the WAG, through the specification 7 that was not verified. To solve the encountered problem, it was necessary to introduce a logout action linked to a page. Other specifications were not verified as, for example the specification concerning the login action (property 8 in the previous section) because of the absence of a link to the HomepageW that could enable to follow a link to a login action. Finally, the model had to be modified in order to satisfy the properties 4 and 11 concerning the error management. After a logout it must be possible to login again, so the model checker system found the absence of an arc to connect the logout state to the HomepageW.

\section{Related Work}

To the best of our knowledge only few works have considered web application analysis; anyway most of them are not based on a formal method approach. We briefly describe the more relevant proposals. Some approaches consider the web similar to a database, hence propose conceptual models of its structure; more recent approaches focus on web applications under a web engineering point of view. A complete review of all the modeling techniques is in [8]. HDM [6] is one of the first model-driven design of hypermedia applications; successive proposals are RMM[15], Strudel [9], Araneus[11] they all build on the HDM model, and support specific navigation constructs. Conallen [2] proposes a UML-based methodology. The main advantage of the method is the possibility to represent all the component of a web application using standard UML notations. The method proposed in [7] is based on a UML model of WA and considers the testing and validation of the developed web system. In [5] the method proposes web application analysis based on queue models. Finally, in [4] the proposed method aims to verify the correct 
use of duplicated pages inside a web constructed using HTML language and ASP code. Once again the method does not consider a formal approach. On the other hand, model checking based on a $\mu-$ calculus language has been used in [3]. The approach does not consider the analysis of dynamic pages. Anyway the model of the web they consider is a graph in which states are pages and transitions between states are hyperlinks in the pages, hence hyperlinks cannot be qualified by properties as we do. Previous approaches considered in [14] propose automata to describe the structure of the links in a hypertext and define a branching temporal logic (hypertext logic) HTL to describe the sequence of transitions between states in the automata. The logic is used to verify the propositions of the temporal logic, but again dynamic pages are not considered.

\section{References}

1. E.M. Clarke, O.M. Grumberg, and D.A. Peled. Model Checking. 1999.

2. J. Conallen. Building Web applications with UML. 2002.

3. L. de Alfaro. Model checking the World Wide Web. pages 77-85, 2001.

4. G. Di Lucca and M. Di Penta. An approach to identify duplicated web pages. In 26th Annual International Computer Software and Applications Conference, pages 481 - 486, Oxford, England, 2002.

5. M. Di Penta, G. Antoniol, G. Casazza, and E. Merlo. Modeling web maintenance centers through queue models. In Fifth European Conference on Software Maintenance and Reengineering, pages 131 - 139, Lisbon, Portugal, 2001.

6. D. Schwabe F. Garzotto, P. Paolini. Hdm - a model-based approach to hypertext application design. ACM TOIS, 11(1):1-26, 1993.

7. P. Tonella F. Ricca. Testing processes of web applications. Annals of software engineering, 14(1):93-114, 2002.

8. P. Fraternali. Tools and approaches for developing data-intensive web applications: a survey. ACM Computing Survey, 31(3):227-263, 1999.

9. J. Kang A.Y. Levy M. F. Fernandez, D. Florescu and D. Suciu. Catching the boat with strudel: experiences with a web-site management system. In ACM - SIGMOD, pages 414-425, 1998.

10. K. L. McMillan. The SMV system, February 1992. http://www.cs.cmu.edu/ modelcheck/smv/smvmanual.r2.2.ps.

11. G. Mecca P.Atzeni and P.Meriado. Design and maintenance of data-intensive web sites. In Proc. of EDBT-98, pages 436-450, 1998.

12. E. Di Sciascio, F M. Donini, M. Mongiello, and G. Piscitelli. Anweb: a system for automatic support to web application verification. In Proc. of SEKE '02, pages 609-616, July 2002.

13. E. Di Sciascio, F.M. Donini, M. Mongiello, and G. Piscitelli. Web Applications Design and Maintenance using Symbolic Model Checking. In Proc. of CSMR '03, pages 63-72, Benevento, Italy, March 26-28 2003. IEEE.

14. P.D. Stotts and J.C. Furuta. Hyperdocuments as automata: verification of trace-based browsing properties by model checking. TOIS, 16(1):1-30, 1998.

15. E. Stohr T. Isakowitz and P. Balasubramanian. Rmm : a methodology for structured hypermedia design. Comm. ACM, 38(8):34-44, 1995. 